\title{
The Measurable and Unmeasurable Visual Benefits of 3D Saline Infusion Sonohysterography
}

Abdel-Gadir A*

Division of Reproductive Medicine and Surgery, Al-Salam International Hospital, Al-Messila Clinics Tower, Port Sayeed Road, Kuwait

\begin{abstract}
Objectives: To test the accuracy of 2D and 3D saline infusion sonohysterography in the diagnosis of endometrial polyps, submucous fibroids, intrauterine adhesions and depth of septae in comparison to hysteroscopic findings.

Materials and methods: 197 women with abnormal uterine bleeding, infertility or recurrent miscarriages were included in the study. A provisional diagnosis was made after 2D saline infusion sonohysterography. This was followed be $3 \mathrm{D}$ volume acquisition and manipulation of the $3 \mathrm{D}$ images to make a final diagnosis. All patients had hysteroscopy during the early follicular phase. Findings during $2 \mathrm{D}$ and $3 \mathrm{D}$ saline infusion were compared to each other and to those obtained during hysteroscopy.

Results: There was no difference regarding the primary diagnosis between the two SIS techniques in relation to the presence or absence of polyps, fibroids, intrauterine adhesions or septae. However, 3D SIS gave better visual impression and clearer documentation of the extent of the intrauterine pathology. This was especially so for the number and distribution of endometrial polyps as well as the extent and distribution of intrauterine adhesions and the depth of septae. These were useful preoperative findings for planning and facilitation of surgery. However, there were no differences between the two ultrasound techniques regarding the number or location of submucous fibroids.

Conclusion: 3D saline infusion sonohysterography when available, could add useful information especially for mapping and documenting the extent of intrauterine pathology. This information could be of great help in counselling patients and facilitating hysteroscopic surgery. However, both SIS techniques failed in diagnosing endometrial micropolyps which were seen only during hysteroscopy.
\end{abstract}

Keywords: Saline infusion; Endometrial micropolyps; Hysteroscopy

\section{Introduction}

2D SIS has been described as an accurate method for the detection of endometrial polyps and submucosal fibroids. It was reported to have a potential of being the first line diagnostic method in women with abnormal uterine bleeding [1]. However, more work was considered to be necessary by the same authors to study the potential of 3D SIS in women of reproductive age. A previous article reported that $3 \mathrm{D}$ reformations improved visualization of the uterine fundus and aided in identification or exclusion of fundal contour abnormalities but did not add value in the detection of endometrial abnormalities [2]. On the other hand, Ludwin et al. [3] reported no significant differences in the diagnostic value between 3D-transvaginal ultrasound (TVS) with 2D-SIS and 3D-SIS or between expert 2D-TVS and 3D-TVS with 2D-SIS. They even questioned the need for endoscopy to diagnose common congenital anomalies because of the high accuracy of these ultrasonographic techniques. Yet again, 3D SIS was reported to allow precise recognition and localization of intrauterine lesions and hysteroscopy would be needed only if 2D and 3D SIS showed no abnormality [4]. The overall sensitivity, specificity, positive predictive value, negative predictive value and accuracy of 2 D SIS was reported to be $87.2,100,100,84.2$, 92.4, and 89.3 and that of 3D SIS was $100,100,86.4$, and 93.6 [5]. The highest accuracy was obtained in diagnosing endometrial polyps and Mullerian anomalies and the least with intrauterine synechiae by the same authors. A trend for higher specificity of 3D SIS with the different uterine pathologies was seen, but it did not reach statistical significance. Another article reported no differences in sensitivity of 3D SIS compared with 2D SIS for the diagnosis of all pathologies, but 3D SIS correlated better with hysteroscopy than 2D SIS. At the same time, specificity for histologic diagnosis appeared to be higher with 3D SIS compared with 2D SIS but larger-scale studies were needed to confirm this finding [6].

Finally, a Cochrane meta-analysis in 2017 [7] revealed no statistically significant differences between 2D SIS and 3D SIS. Additionally, summary sensitivity and summary specificity were higher for 3D SIS, but margins for improvement were limited because 2D SIS was already very accurate. Practically, 2D SIS missed a fibroid or polyp in 9 of 100 cases compared to 3 of 100 with 3D SIS in women who had them. On the other hand, 2D SIS indicated the presence of polyps or fibroids in 4 of 100 women compared to 1 in 100 with 3D SIS when there were none. The authors concluded that both 2D SIS and 3D SIS should be considered alternatives to diagnostic hysteroscopy when intracavitary pathology is suspected in subfertile women and in those with abnormal uterine bleeding.

It seems there was wide agreement regarding the benefits of $2 \mathrm{D}$ and 3D SIS in gynaecological practice. However, there are still some differences regarding the value of these techniques relative to hysteroscopy and relative to each other. In this article I am comparing findings after 2D and 3D SIS with those seen during hysteroscopy in 197 women investigated for abnormal uterine bleeding, infertility and repeated miscarriages.

\section{Materials and Methods}

197 women with abnormal uterine bleeding, infertility or repeated miscarriages were investigated with outpatient SIS followed by hysteroscopic surgery under general anaesthesia. Patients with active or

${ }^{*}$ Corresponding author: Abdel-Gadir A, Division of Reproductive Medicine and Surgery, Al-Salam International Hospital, Al-Messila Clinics Tower, Port Sayeed Road, Kuwait, Tel: 0096522232006; E-mail: prof.gadir@gmail.com

Received February 22, 2019; Accepted March 02, 2019; Published March 09, 2019

Citation: Abdel-Gadir A (2019) The Measurable and Unmeasurable Visual Benefits of 3D Saline Infusion Sonohysterography. J Clin Case Rep 9: 1220. doi: 10.4172/2165-7920.10001220

Copyright: ( 2019 Abdel-Gadir A. This is an open-access article distributed under the terms of the Creative Commons Attribution License, which permits unrestricted use, distribution, and reproduction in any medium, provided the original author and source are credited. 
Citation: Abdel-Gadir A (2019) The Measurable and Unmeasurable Visual Benefits of 3D Saline Infusion Sonohysterography. J Clin Case Rep 9: 1220. doi: $10.4172 / 2165-7920.10001220$

recent pelvic or vaginal infections were not examined till the infections were cleared. The technique was done under aseptic conditions using Genetics Standard intrauterine insemination catheters in the majority of cases. Goldstein sonohysterography catheters (Cook Medical) were used in patients with patulous cervix or suspected intrauterine adhesions. The white acorn reduced fluid back flow as it blocked

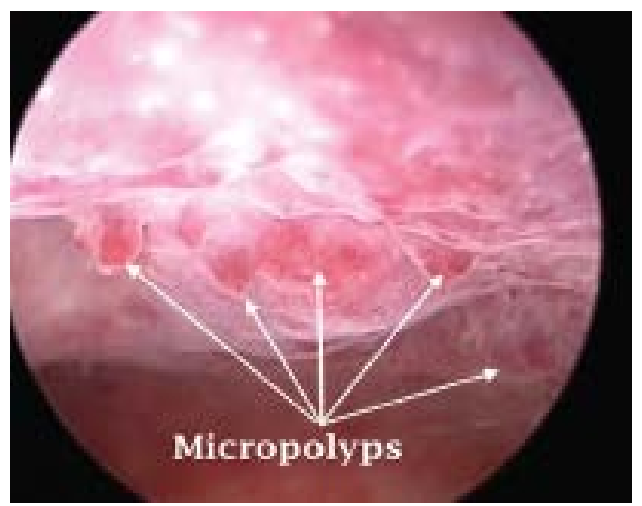

Figure 1: Shows a magnified hysteroscopic image with clusters of micropolyps on the anterior uterine wall. Other similar areas were also seen in other parts of the uterus. Micropolyps are $\leq 1 \mathrm{~mm}$ thick and considered to be reliable signs of chronic endometritis.

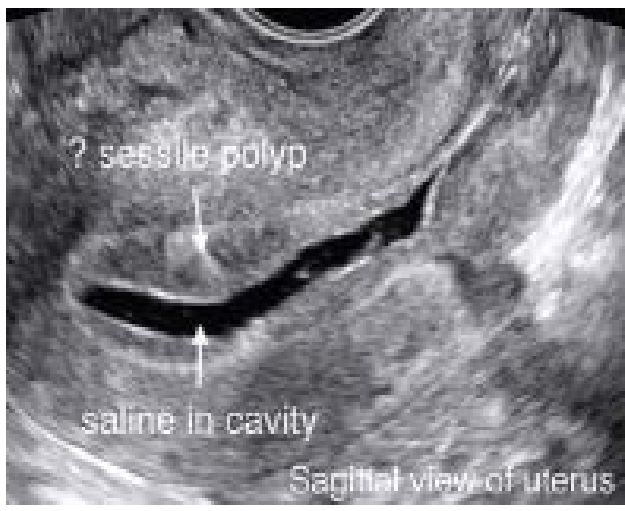

Figure 2: Show a 2D SIS sagittal view of a uterus with unequal thickness of the anterior and posterior endometrial echoes.

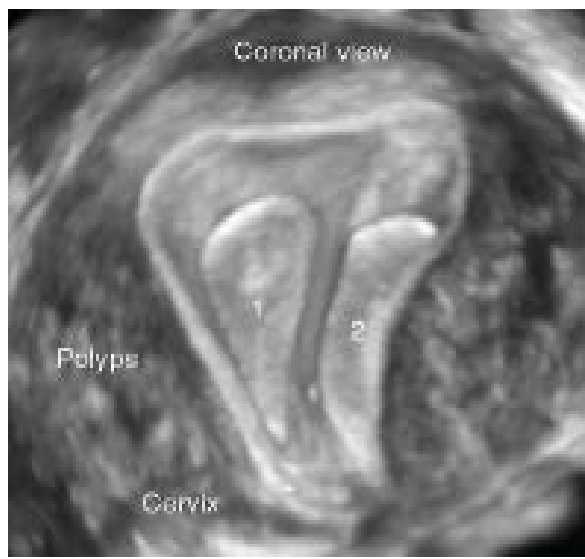

Figure 3: Shows a 3D SIS coronal image of the same uterus shown in Figure 2. It clearly shows two large polyps rising from the lower uterine cavity. The rest of the endometrium looked normal.

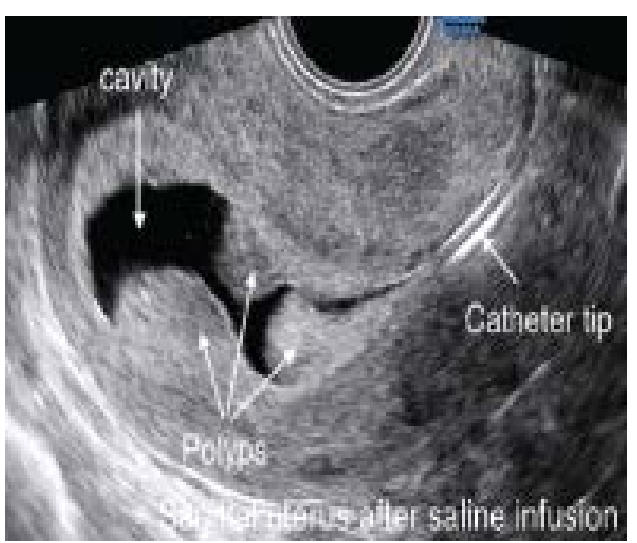

Figure 4: Shows 2D SIS sagittal view of a uterus with 3 identifiable large endometrial polyps.

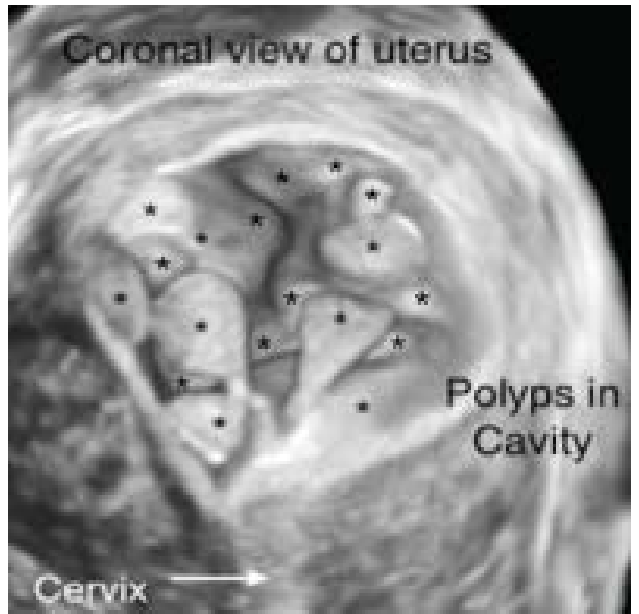

Figure 5: Shows 3D SIS coronal image of the same uterus depicted in Figure 4. It showed 18 different polyps (.) and their exact location within the cavity as confirmed by hysteroscopy. The patient was 46 -years-old at presentation and had two polypectomy procedures during the previous 7 years. She was advised to have a hysterectomy because of her age and the sheer number of polyps but was not agreeable. Transcervical hysteroscopic resection of the polyps and endometrium was done which revealed malignant changes in few polyps after histopathological examination. A hysterectomy was ultimately done, but no residual malignancy was found.

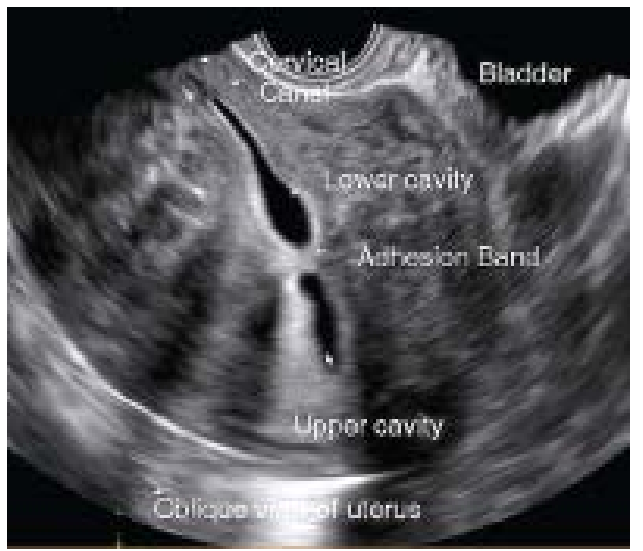

Figure 6: Shows 2D SIS image of a uterus with an adhesion band in the middle dividing the cavity in two compartments. This patient had myomectomy before. 


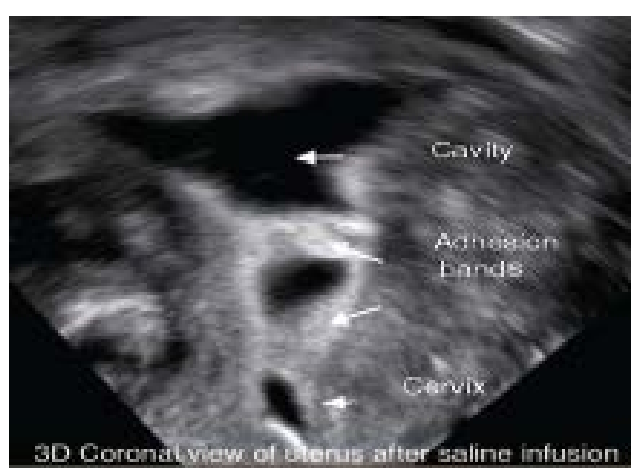

Figure 7: Shows 3D coronal view of the same uterus depicted in Figure 6. It shows the cavity divided in three compartments, rather than two. There is also bright scarring of the endometrium in the lower and mid cavity.

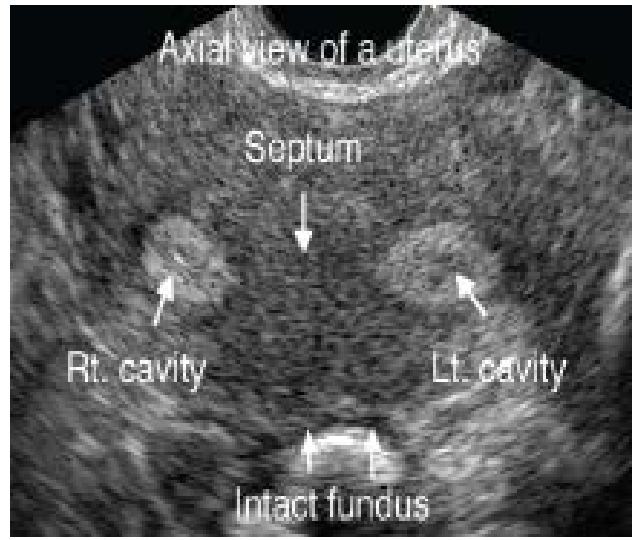

Figure 8: Shows 2D TVS axial view of a uterus during the luteal phase of the cycle. It shows a wide septum separating the upper cavity in two parts. The echogenic luteal phase endometrium facilitated seeing the septum clearly in between. An intact fundus is also clearly seen which excluded the possibility of a bicornuate anomaly.

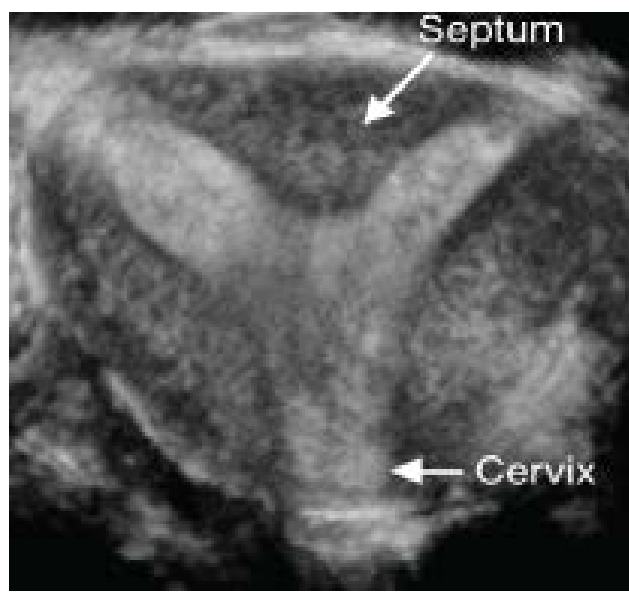

Figure 9: Shows a 3D coronal view of a septate uterus. Note how the depth of the septum is clearly shown. One further advantage shown by this image was the intact endometrial/myometrial interface which excluded the possibility of any sub endometrial adenomyosis. Scanning patients during the mid or late luteal phase of the cycle gave excellent images with the echogenic endometrium against the neighboring myometrium, obviating the need for SIS to diagnose intrauterine septae unless another pathology was suspected. the external os and kept stable intrauterine pressure. Catheters were primed with normal saline before their introduction into the cervical canal to avoid introducing air bubbles into the uterine cavity which might have interfered with the diagnosis. Injuring the endometrial line was avoided by keeping the catheters only in the cervical canal to prevent creation of false pseudopolyps. Saline was instilled slowly into the cavity to reduce the possibility of inciting pain. The best images were obtained in 2D sagittal or oblique planes. An initial provisional diagnosis was made before 3D volumes were then obtained of the same images. The intracavity probe V5-9 was used in all cases mounted on a Samsung Ultrasound Machine, model UGEO WS80A. All patients consented to have the procedures and to use their nonidentifying data for teaching and research. The procedures were always performed for medical indications, as a service under antibiotic cover. Patients were well covered while being examined in the lithotomy position in the presence of a female chaperon. They were asked to report any pain they might have felt as mild, bearable or unbearable. Furthermore, they were assured that both oral and injectable analgesics were available for them on request. Hysteroscopy was done during the early follicular phase in the Day Case Theatre under general anaesthesia.

\section{Results}

None of the patients reported unbearable pain or had an infection after saline infusion. Few patients reported some discomfort during application of the Goldstein catheters. Both SIS techniques did not show any definitive pathology in 37 patients who needed diagnostic hysteroscopy. $18(48.6 \%)$ of these patients showed endometrial micropolyps during hysteroscopic examination (Figure 1). A total number of 81 patients showed micropolyps, 63 of them were combined with other pathologies. None of them was diagnosed as such during ultrasound scan examinations. All other patients with polyps (67 patients), submucous fibroids (41 patients), uterine septae (34 patients), focal subendometrial adenomyosis (11 patients) and intrauterine adhesions ( 7 patients), were $100 \%$ prediagnosed correctly with both SIS techniques as confirmed by hysteroscopic examination. So, except for micropolyps there were no difficulties in reaching a basic diagnosis while using both SIS techniques. However, there was significant difference in the number of diagnosed polyps between the two SIS groups. In 18/67 cases (26.9\%) more polyps were seen during hysteroscopy than prediagnosed with 2 D SIS, compared to only $5 / 76$ (7.5\%) after 3D SIS examination $(\mathrm{p}=0.016)$. Figures 2 and 3 showed sagittal 2D and 3D SIS images respectively of the same uterus. The 2D image showed only a thick endometrium with a suspected sessile polyp on one side. On the other hand, Figure 3 showed a 3D coronal view of the same uterus with two polyps clearly seen rising up from the lower cavity. The endometrium otherwise looked normal. The diagnosis was different, and the management was accordingly different. With thick homogenous endometrium, a progestogen might have been tried first. With endometrial polyps, surgical excision was necessary. This is a good example how 3D SIS changed the line of management.

Figures 4 and 5 respectively showed 2D and 3D views of another uterus. More polyps were seen in the 3D SIS image which allowed better counselling of the patient at the time. Similarly, 3D SIS showed different extent of adhesions compared to the 2D technique in all 7 patients, agreeing with the findings during hysteroscopy. Figure 6 showed 2D SIS image of a uterus with a single band of adhesion across the cavity. On the other hand, Figure 7 showed 3D SIS coronal view of the same uterus with two thick adhesion bands dividing the cavity into three compartments rather than two. In this case operative hysteroscopy was done using a pair of scissors to cut through the less 
dense scar on the right side until the upper cavity was reached. Normal saline was used during this part of the procedure. This was followed by introducing a resectoscope though the same space created with scissors before shaving the two very thick adhesion bands with a monopolar loop electrode. This technique reduced the volume of glycine used. This case is another example to show how 3D SIS superseded the 2D technique in facilitating the surgical procedure. As well, 3D SIS gave better visual impression of the uterine septae depth than 2D SIS. This impression was valid even while using basic $2 \mathrm{D}$ and $3 \mathrm{D}$ techniques without using SIS (Figures 8 and 9). However, there was no difference between 2D and 3D SIS in showing the exact number of submucous fibroids in all cases.

\section{Discussion}

Comparing 2D and 3D SIS techniques showed significant difference between the two in diagnosing the number of endometrial polyps. More polyps were seen with 3D SIS. On the other hand, there was no difference between the two regarding the diagnosis and number of submucous fibroids. This might be due to the small numbers of submucous fibroids in each patient, hence the small scope for error. 3D SIS also gave better visual information about the location and extent of adhesions which made operative hysteroscopy easier in few cases (Figures 6 and 7). It also helped with selecting the route to take into the uterus to excise these lesions. As for intrauterine septae, there were no differences between $2 \mathrm{D}$, and $3 \mathrm{D}$ done during the luteal phase in comparison to the SIS techniques. The echogenic endometrium gave a good contrast against the less echogenic myometrium with good demarcation of the septae (Figures 8 and 9). Accordingly, using SIS for the mere diagnosis of intrauterine septae has been halted in our practice unless another co-pathology was suspected e.g., polyp or fibroid.

It was also evident that neither $2 \mathrm{D}$ nor $3 \mathrm{D}$ SIS were effective in diagnosing micropolyps as isolated findings in 18 cases or when associated with other pathologies in 63 patients. Micropolyps were reported to be significantly associated with endometrial inflammation and were considered reliable diagnostic signs of chronic endometritis [8]. Missing them during ultrasound scanning may lead to unnecessary surgical interventions. It was evident that saline infusion sonohysterography was not very effective in diagnosing small pathologies with equivalent accuracy to diagnostic hysteroscopy. This point was raised in a previous publication [9] and could be secondary to the small size of micropolyps $(\leq 1 \mathrm{~mm}$ ) which might be below ultrasound machines resolution.

\section{Conclusion}

3D SIS added visual advantages related to the number and location of endometrial polyps, the extent of intrauterine adhesions and the depth of intrauterine septae. However, there were no similar advantages in relation to submucous fibroids. It also facilitated planning and performance of quicker hysteroscopic surgery with reduced amount of distension fluid used during the procedures. As all cases with micropolyps were missed by both SIS techniques, diagnostic hysteroscopy would still be necessary for symptomatic patients with negative 2D and 3D SIS findings. This could be done as an office procedure whereas patients with positive SIS findings need to be booked for operative hysteroscopy from the outset.

\section{References}

1. Bittencourt CA, Simoes DSR, Bernardo WM, Fuchs LFP, Soares JM, et al. (2017) Accuracy of saline contrast sonohysterography in detection of endometrial polyps and submucosal leiomyomas in women of reproductive age with abnormal uterine bleeding: Systematic review and meta-analysis. Ultrasound Obstet Gynecol 50: 32-39.

2. Ghate SV, Crockett MM, Boyd BK, Paulson EK (2008) Sonohysterography: Do 3D provide additional value?. AJR Am J Roentgenol 190: 227-233.

3. Ludwin A, Pitynski K, Ludwin I, Banas T, Knafel A (2013) Two-and threedimensional ultrasonography and sonohysterography versus hysteroscopy with laparoscopy in the differential diagnosis of septate, bicornuate and arcuate uteri. J Minim Invasive Gynecol 20: 90-99.

4. Sylvestre C, Child TN, Tulandi T, Tan SLL (2003) A prospective study to evaluate the efficacy of two-and three-dimensional sonohysterography in women with intrauterine lesions. Fertil Steril 79: 1222-1225.

5. Aboulghar MM, Shoeir IK, El-Mohammad MM, Ezzat H (2011) A comparative study of 2-dimensional sonohysterography versus 3-dimensional sonohysterography in infertile patients with uterine cavity lesions and abnormalities. MEFSJ 16: 67-71.

6. Terry S, Banks E, Harris K, Dar P (2009) Comparison P of 3-Dimensional with 2-Dimensional saline infusion sonohysterograms for the evaluation of intrauterine abnormalities. JCU 37: 258-262.

7. Nieuwenhuis LL, Hermans FJR, Bij de Vaate A, Leeflang MMG, Brölmann HAM et al. (2017) Three-dimensional saline infusion sonography compared to twodimensional saline infusion sonography for the diagnosis of focal intracavitary lesions. Cochrane Db Syst Rev p: 5.

8. Cicinelli E, Resta L, Nicoletti R, Zappimbulso V, Tartagni M, et al. (2005) Endometrial micropolyps at fluid hysteroscopy suggest the existence of chronic endometritis. Hum Reprod 20: 1386-1389.

9. Mohammad FH, Fattah AH, Abd-Elrahman AM (2018) Comparative study between three-dimensional transvaginal ultrasonography and hysteroscopy in the diagnosis of uterine cavity abnormalities. EJHM 73: 7350-7357. 